\title{
A quantitative portrait of three xylanase inhibiting protein families in different wheat cultivars using 2D-DIGE and multivariate statistical tools
}

\author{
Evi Croes ${ }^{a}$, Kurt Gebruers ${ }^{a}$, Sebastien Carpentier ${ }^{b}$, Rony Swennen ${ }^{b}$, Johan Robben ${ }^{c}$, \\ Kris Laukens $^{d}$, Erwin Witters ${ }^{d, e}$, Jan A. Delcour ${ }^{a}$, Christophe M. Courtin $^{a, *}$
}

${ }^{a}$ Laboratory of Food Chemistry and Biochemistry, Department of Microbial and Molecular Systems, and Leuven Food Science and Nutrition Research Centre (LFoRCe), Katholieke Universiteit Leuven, Kasteelpark Arenberg 20/2463, B-3001 Leuven, Belgium

${ }^{\mathrm{b}}$ Division of Crop Biotechnics, Katholieke Universiteit Leuven, Kasteelpark Arenberg 13/2455, B-3001 Leuven, Belgium

${ }^{\mathrm{C} B i o c h e m i s t r y, ~ M o l e c u l a r ~ a n d ~ S t r u c t u r a l ~ B i o l o g y ~ S e c t i o n, ~ K a t h o l i e k e ~ U n i v e r s i t e i t ~ L e u v e n, ~ C e l e s t i j n e n l a a n ~ 200 G / 2413, ~ B-3001 ~ L e u v e n, ~ B e l g i u m ~}$

${ }^{\mathrm{d} C e n t e r ~ f o r ~ P r o t e o m e ~ A n a l y s i s ~ a n d ~ M a s s ~ S p e c t r o m e t r y, ~ C a m p u s ~ M i d d e l h e i m, ~ U n i v e r s i t e i t ~ A n t w e r p e n, ~ G r o e n e n b o r g e r l a a n ~ 171, ~}$ B-2020 Antwerpen, Belgium

eFlemish Institute for Technological Research (VITO), Boeretang 200, B-2400 Mol, Belgium

\section{A R T I C L E D A T A}

\section{Article history:}

Received 8 January 2009

Accepted 12 February 2009

\section{Keywords:}

2D-DIGE

Cultivar

Multivariate statistical analysis

Wheat

Xylanase inhibitors

\begin{abstract}
A B S T R A C T
Wheat grains contain three classes of xylanase inhibitors (XIs), i.e. TAXI (Triticum aestivum xylanase inhibitor), XIP (xylanase inhibiting protein) and TLXI (thaumatin-like xylanase inhibitor). These proteins are involved in plant defence and strongly affect cereal-based processes in which inhibitor-sensitive xylanases are used. This paper reports on the successful use of 2D-DIGE and tandem MS to discriminate XI (iso)forms and measures their qualitative and quantitative variation in six different wheat cultivars. In total, 18 TAXI-, 27 XIP- and 3 TLXI-type XI spots were identified. The multiple members of the large TAXI-gene family make a considerable contribution to the total TAXI population. For XIP-type XIs, XIP-I is expressed as the predominant form, albeit under variable degrees of PTMs. Only one TLXI genetic variant was identified, showing different degrees of glycosylation. Multiple comparison analysis revealed up to 5-fold intercultivar differences in protein level of XI (iso)forms. Evaluation of abundance patterns using multivariate statistical tools revealed highly distinctive as well as correlated levels of different XI forms among the six cultivars. As the constitutive (and induced) levels of the different XI (iso)forms, which are differentially regulated in response to various forms of stress in other wheat plant parts, considerably vary between the cultivars, it can be assumed that their degree of resistance against pathogenic attack differs. Similarities in abundance profiles between XI (iso)forms and pathogenesis-related chitinases are also in line with a role in plant defence.
\end{abstract}

(c) 2009 Elsevier B.V. All rights reserved.

Abbreviations: BVA, Biological variation analysis; DIA, Differential in-gel analysis; EDA, Extended data analysis; GH, Glycoside hydrolase; MOWSE, Molecular weight score; PGIP, Polygalacturonase inhibiting protein; PR, Pathogenesis-related; TAXI, Triticum aestivum xylanase inhibitor; TLXI, Thaumatin-like xylanase inhibitor; XI, Xylanase inhibitor; XIP, Xylanase inhibiting protein.

* Corresponding author. Laboratory of Food Chemistry and Biochemistry, Katholieke Universiteit Leuven, Kasteelpark Arenberg 20/2463, B-3001 Leuven, Belgium. Tel.: +32 16321634; fax: +32 16321997.

E-mail address: christophe.courtin@biw.kuleuven.be (C.M. Courtin). 


\section{Introduction}

Plants are continually exposed to pathogens, and hence, have developed several strategies to defend themselves in order to survive. Pathogen attack often starts at the cell wall surface with the production of cell-wall degrading enzymes, such as polygalacturonases and glucanases, by the invading organism. For infection of wheat in particular, endo- $\beta$-D-1,4xylanases (xylanases) are important as the cell wall largely consists of arabinoxylans [1]. In response to attack, plants, among other actions, synthesize pathogenesis-related (PR)proteins, which are involved in plant protection.

In wheat, three structurally different classes of xylanase inhibiting proteins (XIs), showing homologies with PR-proteins, are expressed; i.e. Triticum aestivum xylanase inhibitor (TAXI) [2,3], xylanase inhibiting protein (XIP) $[4,5]$ and thaumatin-like xylanase inhibitor (TLXI) [6]. In light of their specificity towards xylanases of microbial origin $[4,6,7]$, and their inducibility by pathogens and wounding [8-10], XIs are indeed believed to play a role in plant defence, rather than that they have a regulatory function within the plant. Additionally, proteomic [11] as well as transcriptomic studies [9,10] revealed that XIs appear in wheat grains as polymorphic families. Often, plant defence-related proteins are encoded by families of related genes which usually have specific structural and regulatory features [12]. The evolutionary advantage of having multiple genes can derive from their different modes of regulation, which may ensure the expression of at least one gene member in different infection scenarios. In addition, the wheat plant may have evolved a large heterogeneity of XIs to obtain distinct recognition specificities towards the wide variety of xylanases, secreted by pathogenic species. A cycle of adaptation and counter-adaptation between the plant and its enemy has been described for several PR-proteins, including polygalacturonase inhibitors (PGIPs) $[13,14]$. Apart from their biological role, XIs have been mainly studied because of their impact in cereal-based applications [15], such as bread-making [16], gluten-starch separation [17] and refrigerated doughs [18].

In this study, the high resolving power of 2-DE and MALDITOF MS/MS was used to distinguish the different TAXI-, XIP- and TLXI-type XI (iso)forms and to identify them within the complex pattern of the wheat grain proteome. Furthermore, DIGE technology was used to measure intercultivar changes in abundance for these large families of XI forms and to provide an indication of the relative contribution of each of these forms among the total XI population. Up until now, the intercultivar variation in XI levels has only been determined for the 'total' pool of TAXI-, XIP- or TLXI-type XIs using xylanase inhibition activity assays [19,20] and/or immunoblot quantification methods [21]. Here, for the first time, we provide an in-depth analysis of the complexity of XI proteins in different wheat cultivars. Multivariate statistical tools were used to reveal either remarkable similarities or differences in abundance profiles within each class of XIs, between different classes of XIs as well as between XIs and non-XI proteins, with an emphasis on plant defencerelated proteins. This sheds some light on possible relationships in gene expression and post-translational regulation within different wheat cultivars and provides additional information on the biological function(s) of the different (iso)forms.

\section{Materials and methods}

\subsection{Materials}

Wheat (T. aestivum) cultivars Glenlea, Lona, Klein-Estrella, Martonvasari-17, Kirkpinar-79, Hereward (harvest 2005) were obtained from Dr. Zoltan Bedo (Agricultural Research Institute of the Hungarian Academy of Sciences, Martonvasar, Hungary) and ground into wholemeal using a Perten SKCS 3100 laboratory mill (Huddinge, Sweden). The six cultivars were selected for their distinct TAXI, XIP and TLXI levels, based on xylanase inhibition activity measurements and/or immunoblotting and probing with anti-XI specific polyclonal antibodies [21]. All chromatographic and electrophoretic media were obtained from GE Healthcare (Uppsala, Sweden). Complete Protease Inhibitor Cocktail tablets were from Roche Diagnostics (Vilvoorde, Belgium). Other chemicals and reagents were purchased from Sigma-Aldrich (Bornem, Belgium) and were of analytical grade unless specified otherwise.

\subsection{Experimental setup, sample preparation and protein labelling}

Wheat grain extracts of the six selected wheat cultivars were prepared in quadruplicate as described previously [11]. Briefly, samples were crushed in liquid nitrogen and $250 \mathrm{mg}$ was suspended in $1.0 \mathrm{ml}$ Tris-HCl buffer (50.0 mM, pH 7.8), containing Complete Protease Inhibitor Cocktail tablets (1 tablet/10 $\mathrm{ml}$ extraction buffer). Soluble wheat seed proteins were precipitated with 4 volumes $10 \%$ TCA in acetone (overnight, $-20{ }^{\circ} \mathrm{C}$ ) and pellets were washed twice in $80 \%$ acetone and air-dried. Precipitated proteins were resuspended in labelling buffer (7.0 M urea, 2.0 M thio-urea, 4.0\% CHAPS, $30 \mathrm{mM}$ Tris). Where necessary, the $\mathrm{pH}$ was adjusted to $\mathrm{pH}$ 8.5. Protein concentrations were determined using the 2D-Quantkit (GE Healthcare). In general, minimal labelling with $\mathrm{N}$ hydroxysuccinimidyl-ester dyes Cy-3, Cy-5 and Cy-2 (GE Healthcare) was performed as described by the manufacturer. In brief, for each biological replicate, $50 \mu \mathrm{g}$ of protein was incubated (30 min, on ice, in the dark) with $357 \mathrm{pmol}$ of Cy-3 or Cy-5, freshly dissolved in anhydrous dimethyl formamide. The reaction was quenched by the addition of $1.0 \mu \mathrm{l}$ and $10 \mathrm{mM}$ lysine (10 $\mathrm{min}$, on ice, in the dark). Reciprocal labelling, in which half of the replicates of each cultivar were labelled with Cy-3 and the other half with Cy-5, was applied. This way, artificial effects due to preferential labelling were avoided. In addition, a pooled internal standard, consisting of $25 \mu \mathrm{g}$ of each of the 24 samples, was labelled with Cy-2. Equal volumes of labelling buffer, supplemented with $40 \mathrm{mM}$ DTT and $1.0 \%$ IPG-buffer pH 6-11, were added to the labelled samples. Cy-3 and Cy-5 labelled replicates (24) were randomly grouped and mixed with the Cy-2 labelled internal standard (12).

\subsection{2-DE and image analysis}

Labelled wheat grain albumins/globulins were separated by 2$\mathrm{DE}$ as described previously [11]. In short, after rehydration in Destreak rehydration solution (GE Healthcare) containing $0.5 \%$ IPG-buffer $\mathrm{pH} 6-11$, isoelectric focusing $\left(\sim 30 \mathrm{kVh}, 20^{\circ} \mathrm{C}\right)$ was 
performed on $18 \mathrm{~cm}$ IPG-strips in a pI range of 6 to 11 using the Ettan IPGphor II IEF unit (GE Healthcare). SDS-PAGE was carried out at $20^{\circ} \mathrm{C}$ on $15.0 \%$ homogenous polyacrylamide gels (MM range of 10 to $60 \mathrm{kDa}$ ) using the Ettan Daltsix vertical electrophoresis system (GE Healthcare) in conjunction with the Tris-glycine buffer system [22]. The differentially labelled co-resolved protein maps were imaged using a Typhoon 9400 scanner (GE Healthcare) at dye-specific excitation wavelengths and emission band filters at $100 \mu \mathrm{m}$ resolution.

Four preparative gels, containing $400 \mu \mathrm{g}$ of wheat grain albumins/globulins, were run in parallel with the DIGE gels and were stained with Sypro Ruby protein stain (Invitrogen, Carlsbad, CA, USA) according to the manufacturers' instructions.

\subsection{Statistical analysis}

Images were properly cropped and exported for analysis in DeCyder software version 6.5 (GE Healthcare). They were first processed using the DeCyder DIA (Differential In-gel Analysis) software module to detect and differentially quantify the protein spots in images from the same gel. Calculated volume ratios Cy-3:Cy-2 and Cy-5:Cy-2 of all individual protein spots were imported in the DeCyder BVA (Biological Variation Analysis) software module. Gel-to-gel matching of the standard protein maps was then performed, followed by statistical analysis based on the standardized protein log abundances. ANOVA was applied to the matched spots to test for differences in protein levels among the cultivars. Using the EDA Version 1.0 (Extended Data Analysis) module within the DeCyder software version 6.5 (GE Healthcare), data were processed using multivariate analysis. Initially, a principal component analysis (PCA) was performed on the proteins occurring in at least $75 \%$ of all the spot maps to identify sample outliers. A multiple comparison test $(P<0.01)$ was performed to detect the significantly different wheat cultivars. To determine the intra-XI quantitative ratios, the volumes of the identified spots were exported to perform Tukey's tests $(P<0.05)$ with the Statistical Analysis System software 8.1.

\subsection{Protein identification}

Spots of interest were excised from four preparative gels using the Ettan Spot Picker (GE Healthcare). For MS analysis, spots were collected in microtiter plates and dehydrated gel particles were incubated on ice submerged in $2.0 \mu$ ligest buffer containing 25 ng trypsin (Promega MS Gold, Promega, Madison, WI, USA), $100 \mathrm{mM} \mathrm{NH}_{4} \mathrm{HCO}_{3}$ and $10 \% \mathrm{CH}_{3} \mathrm{CN}(\mathrm{v} / \mathrm{v})$. After $30 \mathrm{~min}$ of rehydration, an additional $10 \mu \mathrm{l}$ of a buffer containing $25 \mathrm{mM}$ $\mathrm{NH}_{4} \mathrm{HCO}_{3}$ and $10 \% \mathrm{CH}_{3} \mathrm{CN}(\mathrm{v} / \mathrm{v})$ was added. After $180 \mathrm{~min}$ of proteolysis at $37{ }^{\circ} \mathrm{C}$, the resulting peptides were concentrated and desalted using micro column solid phase tips (PerfectPureTM C18 tip, 200 nl bed volume, Eppendorf, Hamburg, Germany) and eluted directly onto a MALDI target plate (OptiMaldi plate, Applied Biosystems, Foster City, CA, USA) using a $1.1 \mu$ l volume of $50 \%(\mathrm{v} / \mathrm{v}) \mathrm{CH}_{3} \mathrm{CN}: 0.1 \%(\mathrm{v} / \mathrm{v}) \mathrm{CF}_{3} \mathrm{COOH}$ solution saturated with recrystallized $\alpha$-cyano-4-hydroxycinnamic acid and spiked with internal standards, i.e. $20 \mathrm{fmol} / \mu \mathrm{l}$ Glu1-Fibrinopeptide B $(\mathrm{m} / \mathrm{z}$ 1570.677), des-Pro2-Bradykinin ( $\mathrm{m} / \mathrm{z}$ 963.516) and Adrenocorticotropic Hormone Fragment 18-39 Human ( $\mathrm{m} / \mathrm{z}$ 2465.198). A MALDI-tandem MS instrument (MALDI TOF/TOF 4800, Applied
Biosystems) was used to acquire PMFs and subsequent $1 \mathrm{kV}$ CID fragmentation spectra of selected peptides. PMF spectra and peptide sequence spectra were obtained using the settings as presented in Supplementary Tables S1, S2. Each MALDI plate was calibrated according to the manufacturers' specifications. All PMF spectra were internally calibrated using the standards ( $\pm 10 \mathrm{ppm}$ threshold). This resulted in an average mass accuracy of 5 ppm of any analyzed peptide spot. Using the individual PMF spectra, up to eight peptides exceeding an $\mathrm{S} / \mathrm{N}$ value of 40 that passed through a mass exclusion filter (Table S3) were submitted to fragmentation analysis. Fragmentation spectra were recorded according to the settings displayed in Table S2 (no internal calibration was used). PMF spectra and the peptide sequence spectra of each sample were processed with the accompanied software suite (GPS Explorer 3.5, Applied Biosystems) and parameters were set as summarized in Table S4. Data search files were generated according to settings presented in Table S5 and submitted against non-redundant NCBI restricted to Viridiplantae and complemented with all Genbank plant XI sequences (Table 2) as well as clustered XI-encoding EST sequences for protein homology based cross-species identification $[23,24]$ using a local database search engine (Mascot 2.1, Matrix Science). Retained hits have molecular weight search (MOWSE) scores (falling within the 95th percentile confidence interval) above 69 [25]. In order to estimate the false positive rate of the protein homology dataset, a decoy database was generated. Each protein amino acid sequence was shuffled using the EMBOSS shuffle tool [26]. Estimation of the false positive rate resulted in the detection of two false positives/1000 queries for $P \leq 0.05$ settings or zero false positives/1000 queries for $P \leq 0.02$ settings.

\section{Results}

\subsection{D-DIGE of seed proteins for six different wheat cultivars}

2-DE and MS analysis of XI proteins, purified from wheat wholemeal on the basis of their affinity for specific xylanases, previously revealed the existence of polymorphic families of TAXI-, XIP-, and TLXI-type proteins [11]. The aim of this study was to differentiate between the isoforms and PTMs of the three types of XIs within a crude wheat grain extract and to elucidate their quantitative variation among different wheat cultivars. Therefore, six wheat cultivars, grown on the same location and in identical environmental conditions, were selected for analysis using 2D-DIGE. The cultivars were chosen because of their distinct 'total' TAXI, XIP, and TLXI levels (Table 1) [21].

Table 1 - 'Total' TAXI, XIP and TLXI levels (ppm) in wholemeal of six wheat cultivars, measured by immunoblotting and densitometric analysis

\begin{tabular}{lccc} 
Cultivar & TAXI $(\mathrm{ppm})$ & XIP $(\mathrm{ppm})$ & TLXI $(\mathrm{ppm})$ \\
\hline Glenlea & 190 & 208 & 124 \\
Lona & 81 & 228 & 84 \\
Klein-Estrella & 179 & 371 & 143 \\
Martonvasari-17 & 111 & 175 & 119 \\
Kirkpinar-79 & 121 & 276 & 150 \\
Hereward & 106 & 156 & 51 \\
\hline
\end{tabular}


Over one thousand soluble wheat seed proteins were resolved in a pI range of 6 to 11 and in a molecular mass range of 10 to $60 \mathrm{kDa}$, a region specifically suited for separation of the three types of XIs. TAXI-type XIs simultaneously occur as a $40 \mathrm{kDa}$ single polypeptide and as a processed form consisting of two disulfide-linked polypeptides of $\sim 10$ and $\sim 30 \mathrm{kDa}$. Only the $10 \mathrm{kDa}$ C-terminal polypeptides of the processed form were lost in these analyses given their more acidic pI values (theoretical pI values of 5.0-5.3). As four biological replicates were analyzed for each cultivar, in total, 36 spot maps were recovered after 2-DE, of which 12 internal standards. After matching, 583 protein spots were found in at least 3 out of 4 spot maps and were included in the PCA. The 24 spot maps discretely clustered into six groups, corresponding to the six cultivars under study (Fig. 1a). The PCA plot thus indicates a high reproducibility between replicate samples and shows distinct protein abundance patterns between the six cultivars. As no outlier spot maps were observed, all of them were incorporated in the further statistical analysis.

\subsection{Identification of XI proteins}

The first issue addressed was the identification of all XI (iso) forms within the complex pattern of wheat seed proteins (Fig. 2). On the basis of previously obtained 2-DE fingerprints of affinity-purified XIs on the one hand and, evaluation of 2Dimmunoblots of complex wheat seed patterns after probing with anti-TAXI, anti-XIP or anti-TLXI antibodies on the other hand [11], regions of interest were defined for which as many spots as possible were excised for MALDI-TOF/TOF analysis. In total, 48 spots were identified as one of the three types of XIs, of which 18 TAXI (11 non-processed $40 \mathrm{kDa}$ forms and 7 cleaved $30+10 \mathrm{kDa}$ forms), $27 \mathrm{XIP}$ and 3 TLXI (iso)forms (Table 3, Table S6). Fig. 2 shows a representative spot map of wheat seed proteins (cultivar Klein-Estrella, Cy-5) for which all identified XI spots are labelled by their master spot ID.

The high amino acid sequence similarity among the different TAXI variants and hence, the limited number of variant specific tryptic peptides, highly complicated the proper assignment of MS spectra to a certain variant (Table S6). Nevertheless, minimally 4 out of 12 TAXI variants, present in our customized database (Table 2), were distinguished for the non-cleaved form of TAXI-type proteins: TAXI-725ACCN, TAXI-Ia, TAXI-IIa and TAXI-IV. Their pIs and molecular masses range from 7.6 to 8.6 and from 38.8 to $40.3 \mathrm{kDa}$, respectively. All these variants were identified in the cleaved form as well, except for TAXI-IIa. The $30 \mathrm{kDa}$ polypeptides have pIs and molecular masses between 8.6 and 9.0, and 26.9 and $30.5 \mathrm{kDa}$, respectively. These findings are in agreement with previous results on affinity-purified XIs [11]. The occurrence of the TAXI-725ACC variant cannot be excluded since this variant shows $97 \%$ identity with the TAXI-725ACCN variant [11]. All tryptic peptides could be accounted for as originating from both forms. The same was true for TAXI-IV and TAXI-IIb, which share $99 \%$ identity [27]. For several spots not all tryptic peptides could be matched to a single TAXI variant, indicating the presence of at least two isoforms in a single spot. Moreover, the same genetic variant could be identified in several spots (Table S6). These observations can be explained by the formation of charge-altering PTMs. Remarkably, all the $40 \mathrm{kDa}$ TAXI spots can be divided in either of two groups, the first group (a) Score plot (spot maps)

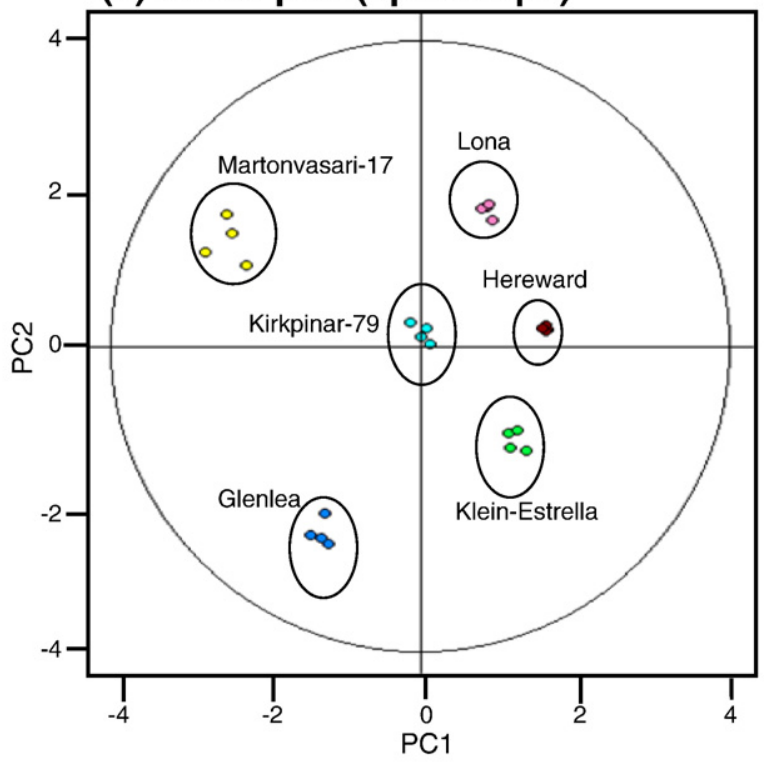

(b) Loading plot (proteins)

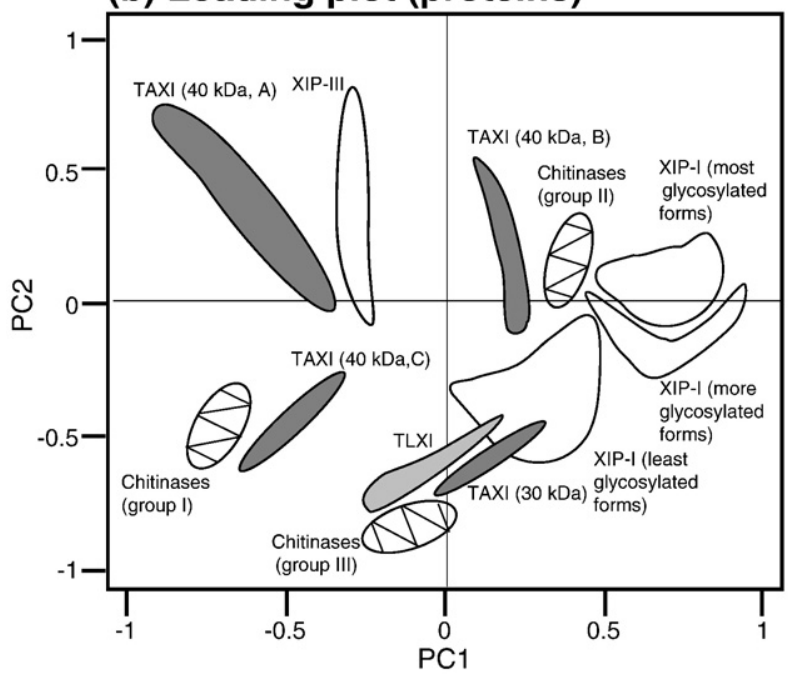

Fig. 1 - (a) PCA plot (score plot) of six cultivars, based on all spots present in at least $75 \%$ of all spot maps. The two principal components discretely cluster the 24 individual spot maps (4 replicates/cultivar) into six reference groups, corresponding to the six cultivars under study. (b) Schematic overview of PCA plot (loading plot) of the identified XIs and chitinases, clustered into groups comprising the following spots (IDs as indicated in Figs. 2 and 5): TAXI (40 kDa, A), spots $346,358,365,370,374,375$; TAXI (40 kDa, B), spots 334 , 340, 347; TAXI (40 kDa, C), spots 330, 339; TAXI (30 kDa), spots 636, 669; XIP-I (least glycosylated forms), spots 706, 709, 711, 713, 714, 716, 717, 720, 722, 882; XIP-I (more glycosylated forms), spots 674, 676, 677, 678, 679; XIP-I (most glycosylated forms), spots 635, 637, 639, 642, 643; XIP-III, spots 624, 659, 689, 700; TLXI, spots 1017, 1049, 1055; chitinases (group I), spots $570,571,586,587$; chitinases (group II), spots 726,750 , 759, 760, 773; chitinases (group III), spots 753, 762, 763, 779. 


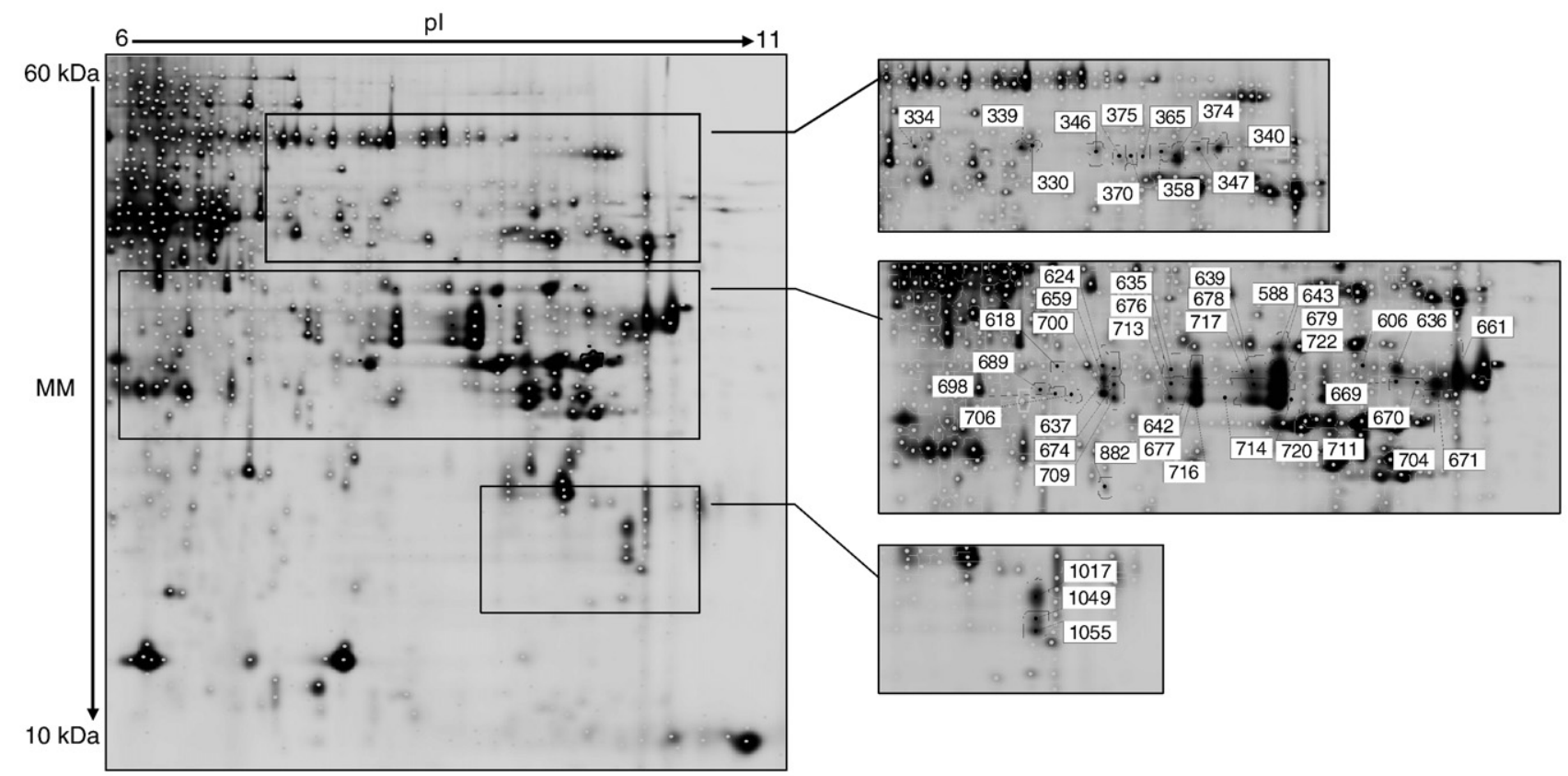

Fig. 2 - Representative spot map of wheat seed albumins/globulins (cultivar Klein-Estrella, Cy-5) in a pI range of 6 to 11 and a molecular mass range of 10 to $60 \mathrm{kDa}$. The positions of the identified XI protein (iso)forms are indicated with their master spot ID in the three zoomed-in regions of the gel.

including 7 spots identified as TAXI-Ia and/or TAXI-725ACCN/ TAXI-725ACC and the second group containing 4 spots identified as TAXI-IV/TAXI-IIb and/or TAXI-IIa. For the processed forms of TAXI-type XIs, the identification was obscured by the presence of highly abundant wheat proteins; i.e. globulin-1 (gil 110341795) or tritin (gi|391929) (Table S6).

The 27 spots identified as XIP-type XIs can mainly be divided into two groups, either containing the XIP-I or the XIPIII variant. The latter was detected in spots 624, 659, 689 and 700 situated near neutral pI, which corresponds to its theoretical PI of 6.9. Tryptic peptides of XIP-I were distinguished in the remaining 24 spots. This implies that both genetic variants are present as different forms, varying in $\mathrm{pI}$ and molecular mass. Vertical shifts were already ascribed to different degrees of glycosylation, while charge heterogeneity is not yet clarified [11]. The identification of XIP-type proteins was highly consistent with earlier results, with the exception that the occurrence of an additional genetic variant XIP-R2 was confirmed in spot 711, next to the XIP-I variant (Table S6). Migration of XIP-R2 to a more alkaline position within the gel is in agreement with its theoretical pI of 8.5.

Finally, three spots corresponded to a single genetic variant of TLXI. They only vary in molecular mass, which is accounted for by differences in glycosylation [11].

\subsection{Relative contribution of different XI (iso)forms to the total XI population}

To determine the relative abundance of each XI form, spot volumes of the identified XI spots of the 24 spot maps were

\section{Table 2 - Putative XI sequences (Triticum aestivum) included in the search database.}

\begin{tabular}{|c|c|c|c|c|c|c|}
\hline \multirow[t]{2}{*}{ Protein name } & \multirow[t]{2}{*}{ Accession no. } & \multicolumn{2}{|c|}{ Theor. MM/pI } & \multirow[t]{2}{*}{ Protein name } & \multirow[t]{2}{*}{ Accession no. } & \multirow[t]{2}{*}{ Theor. MM/pl } \\
\hline & & $40 \mathrm{kDa}$ form & $30 \mathrm{kDa}$ form & & & \\
\hline \multicolumn{4}{|c|}{ TAXI-type xylanase inhibitors } & \multicolumn{3}{|c|}{ XIP-type xylanase inhibitors } \\
\hline TAXI-Ia & gi|116666775 & $38.8 / 8.2$ & 26.9/8.7 & XIP-I & gi|31615809 & $30.3 / 8.3$ \\
\hline TAXI-725ACCN & gi|156186245 & $39.1 / 7.6$ & $27.0 / 8.6$ & XIP-III & gi|66766322 & $30.5 / 6.9$ \\
\hline TAXI-725ACC & gi|156186243 & $39.3 / 8.0$ & $27.2 / 8.3$ & XIP-R1 & gi|153918942 & $30.0 / 7.6$ \\
\hline TAXI-IIa & gi|62996368 & $40.3 / 8.4$ & $27.1 / 9.3$ & XIP-R2 & gi|153918944 & $29.9 / 8.5$ \\
\hline TAXI-Ib & gi|62996372 & $39.2 / 8.6$ & 27.3/9.0 & XIP-II (Triticum durum & gi|18693099 & $30.7 / 9.1$ \\
\hline TAXI-Iib & gi|62996370 & $40.3 / 8.4$ & $27.4 / 8.7$ & & & \\
\hline TAXI-III & gi|56201270 & $39.2 / 8.6$ & $27.3 / 9.0$ & \multicolumn{3}{|c|}{ TLXI-type xylanase inhibitors } \\
\hline TAXI-IV & gi|56201272 & $39.7 / 8.6$ & $27.0 / 9.0$ & TLXI & gi|110836641 & $15.6 / 8.4$ \\
\hline TAXI-801NEW & gi|156186253 & $40.2 / 9.0$ & $27.1 / 9.3$ & & & \\
\hline TAXI-602OS & gi|156186249 & $41.7 / 9.5$ & - & & & \\
\hline TAXI-801OS & gi|156186251 & $46.1 / 9.1$ & - & & & \\
\hline TAXI-725OS & gi|156186247 & $41.6 / 6.0$ & - & & & \\
\hline
\end{tabular}


exported from the DeCyder software. For each spot map separately, volume ratios of individual TAXI, XIP or TLXI spots over the sum of the total population of TAXI, XIP or TLXI spots, respectively, were calculated. This way, the relative contribution of each XI (iso)form to the total level of XIs of a specific inhibitor class was obtained for each wheat cultivar (\% $\mathrm{XI}_{\text {(cultivar) }}$ ) as was the average for the six cultivars (\% $\mathrm{XI}_{\text {(average) }}$ ) using the following formulas:

$\% \mathrm{XI}($ cultivar $)=\frac{\left(\sum_{j=1}^{4}\left(\frac{\text { Volume }[\mathrm{XI}(\mathrm{ID})] \mathrm{i}}{\sum_{i=1}^{\mathrm{XI} \text { spots }} \text { Volume }[\mathrm{XI}(\mathrm{ID})] \mathrm{i}}\right)_{j} \times 100\right)}{4}$

$\mathrm{XI}(\mathrm{ID}$ ): individual XI (iso)form per inhibitor class (spot ID, as indicated in Fig. 2), i: total number of identified XI spots per inhibitor class, $j$ : quadruplicate samples for each cultivar

$\% \mathrm{XI}($ average $)=\frac{\sum_{k=1}^{6} \% \mathrm{XI}(\text { cultivar })_{k}}{6}$

$k$ : averaged over six wheat cultivars.

These calculations were not feasible for the processed form of TAXI-type proteins since most of these spots show a great overlap with non-XI proteins. Fig. 3 shows the percentages of the XI spots, on average for the six cultivars (\% $\left.\mathrm{XI}_{\text {(average) }}\right)$, for (a) TAXI- (40 kDa polypeptides), (b) XIP- and (c) TLXI-type XIs, based on Tukey's tests $(P<0.05)$.

For the $40 \mathrm{kDa}$ polypeptides of TAXI-type XIs the two most intense spots, i.e. spot 340 (TAXI-IV/TAXI-IIb) and spot 374 (TAXI-Ia) (Fig. 2), represent a considerable proportion ( $45 \%$ ) of the total population. As mentioned earlier, when a mixture of two genetic variants was identified for the TAXI spots, each time a combination of TAXI-Ia and TAXI-725ACCN/TAXI725ACC or of TAXI-IV/TAXI-IIb and TAXI-IIa was found. Cultivars differ in the relative proportions of both groups [Fig. 4a, Tukey's test $(P<0.05)$ ]. While cultivars Kirkpinar-79, Klein-Estrella and Martonvasari-17 contain higher levels (6472\%) of the TAXI-Ia/TAXI-725ACCN/TAXI-725ACC group than the TAXI-IV/TAXI-IIb/TAXI-IIa forms, cultivars Lona, Glenlea and Hereward have approximately equal levels of both. The common occurrence of specific TAXI isoforms in one of the two groups is consistent with their chromosomal location and their suggested relationship (Gert Raedschelders, Laboratory of Gene Technology, Katholieke Universiteit Leuven, Leuven, Belgium, personal communication). Indeed, TAXI-Ia and TAXI-725ACCN/TAXI-725ACC on the one hand, and TAXI-IIa and TAXI-IIb/TAXI-IV on the other hand, are believed to be homoeologs, being located on separate genomes of hexaploid wheat. In contrast, for the B-genome specific TAXI-Ia and (a)

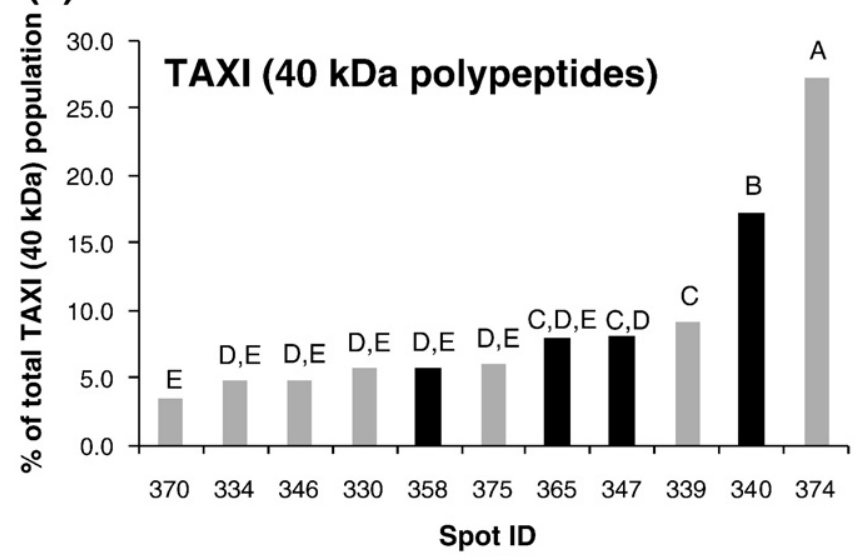

(b)

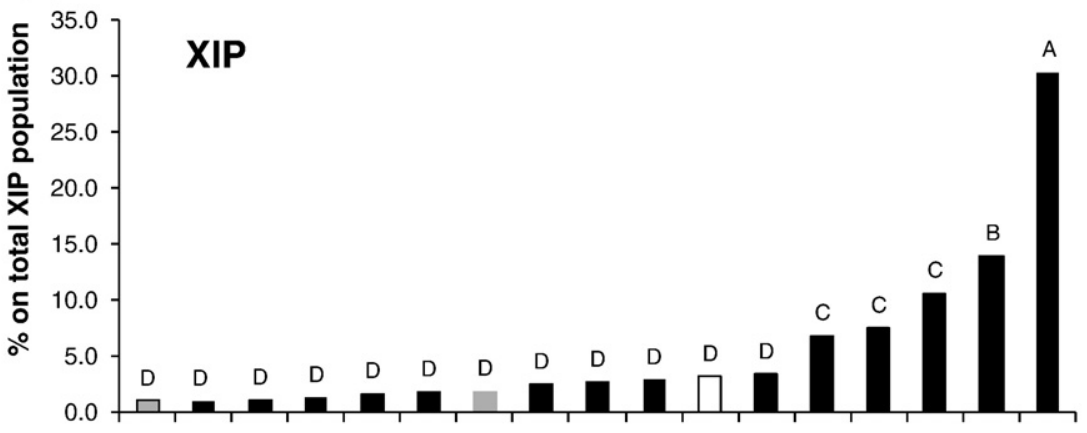

$659720635709639678700642713588711 \quad 677 \quad 717 \quad 716 \quad 643 \quad 679722$

Spot ID (c)

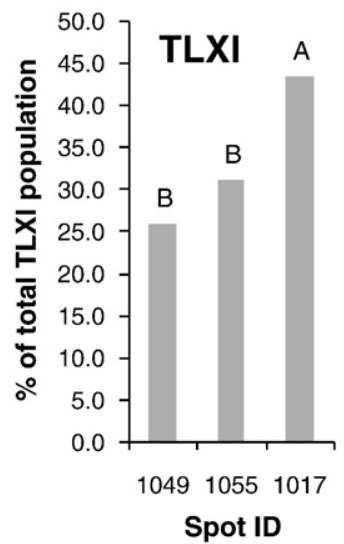


TAXI-IIa, a paralogous relationship is suggested, as is also the case for the D-genome specific TAXI-725ACCN/TAXI-725ACC and TAXI-IIb/TAXI-IV. Generally, the promoter regions of orthologs contain similar regulatory motifs, while this is not necessarily true for paralogs [28]. These findings may also hold for homoeologs and paralogs in polyploid organisms. Fig. 5 shows a phylogenetic tree of all putative TAXI genetic variants.

For XIP-type XIs, on average, the three spots with the highest spot volumes (722, 679 and 643; XIP-I) (Fig. 2) make up approximately $55 \%$ of the total XIP population (Fig. $3 b$ ). In contrast to TAXI-type XIs, a single genetic variant, XIP-I, is predominant, representing on average $92 \%$ of the total level of XIP-type XIs. XIP-III and XIP-R2 occur in much lower levels. An important feature of XIP-type XIs is their glycosylation pattern. Overall, for the multiple (iso)forms, varying in $\mathrm{pI}$, vertical trains, mostly consisting of three separate spots, are visible (Fig. 2). A comparison of the glycosylation degree [11] of the six cultivars reveals that the least or non-glycosylated forms make up the largest part (40-64\%) of the total XIP population [Fig. 4b, Tukey's test $P<0.05)$ ]. The level of the more and the most glycosylated forms is very alike, ranging from $17-28 \%$ and $13-29 \%$, respectively.
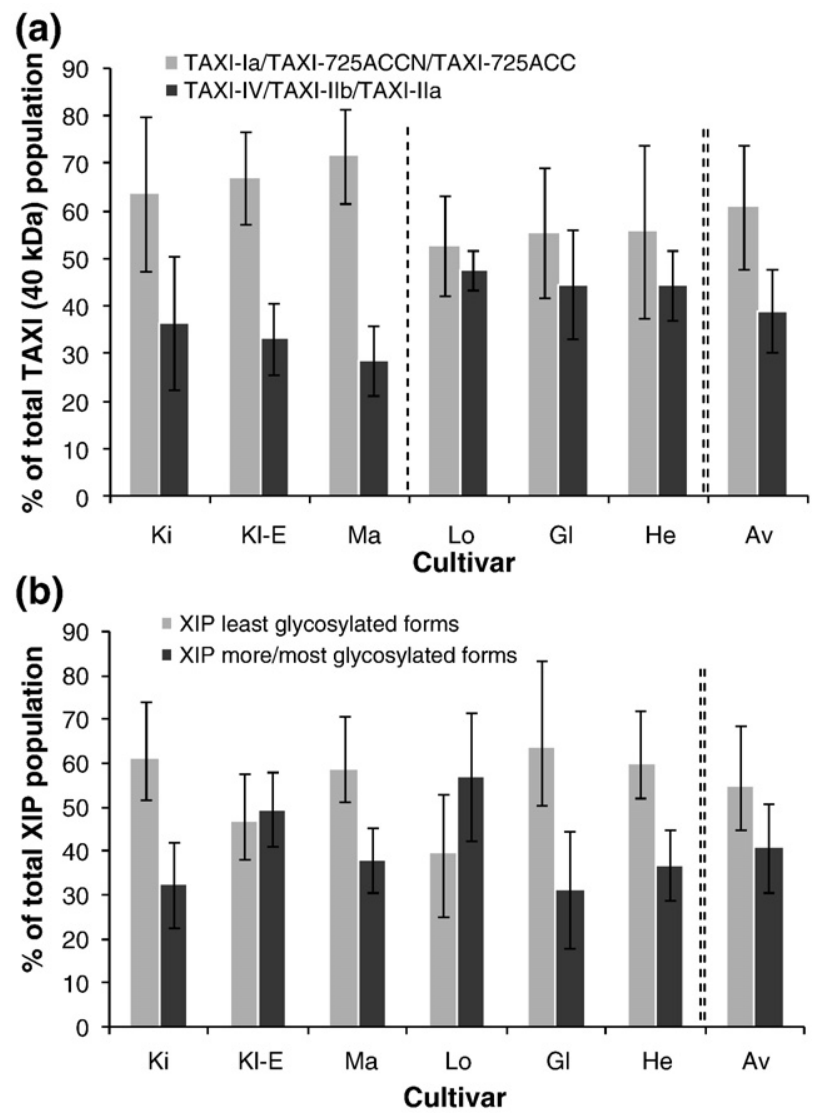

Fig. 4 - (a) Relative contribution of ( ) TAXI-725ACCN/TAXI725ACC/TAXI-Ia and ( ) TAXI-IV/TAXI-IIb/TAXI-IIa to the total TAXI population in six wheat cultivars. (b) Relative contribution of the () least and $(\square)$ more/most glycosylated forms of XIP-type XIs to the total XIP population in six wheat cultivars; Ki: Kirkpinar-79, Kl-E: Klein-Estrella, Ma: Martonvasari-19, Lo: Lona, Gl: Glenlea, He: Hereward, Av, average of six cultivars.

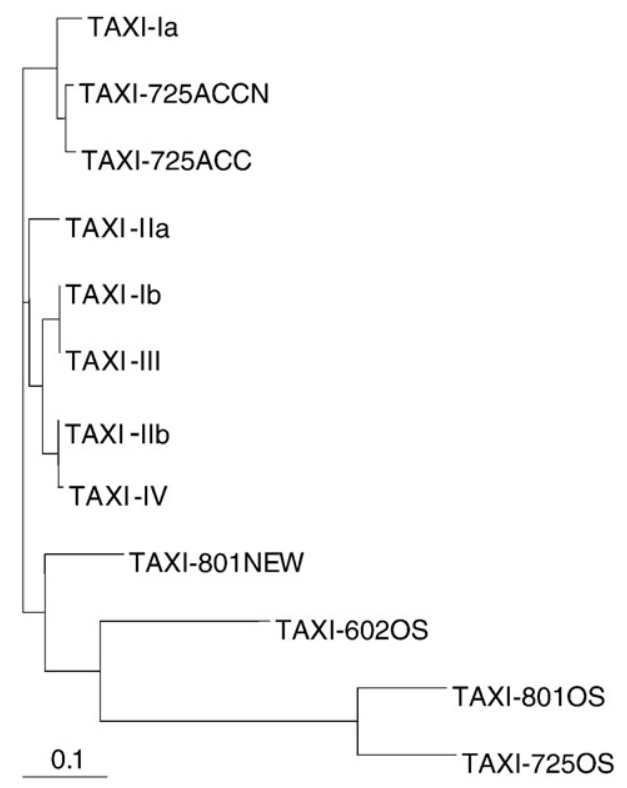

Fig. 5 - Phylogenetic tree showing the relationship between (putative) TAXI sequences. The tree was generated using TreeView 1.6.6.

The opposite was found for TLXI-type XIs, for which only one genetic variant was found. The most glycosylated form (spot 1017, Fig. 2) [11], on average, represents the highest proportion ( $43 \%$ ) of the total TLXI population (Fig. 3c).

\subsection{Intercutivar variation in three classes of polymorphic} XIs

Table 3 represents differences in protein abundance of the large families of XI forms between the six cultivars. One-way ANOVA indicates whether an overall statistically significant difference (threshold $P<0.01$ ) in protein abundance is measured between the six cultivars. The multiple comparison test, in which all cultivars are compared two-by-two, was performed to reveal more specifically for which cultivars the differences in abundance (expressed as average ratios in Tables 3 and S7) are significant. Differences in relative XI levels among the different cultivars are represented in log standard abundance profiles, included in Table 3.

3.4.1. Intercultivar variation within a single class of XIs For the $40 \mathrm{kDa}$ polypeptides of TAXI-type proteins only 5 of the 11 identified spots show significant intercultivar variation. While overall TAXI levels in the six cultivars vary by a factor 2.4 [21], significant differences in the levels of specific TAXI $40 \mathrm{kDa}$ forms range from 1.5 to 4.0. Spot 374, identified as TAXIIa, shows a 4.0- and 3.3-fold higher abundance in cultivar Martonvasari-17, than in Kirkpinar-79 and Hereward, respectively. As this TAXI-form, on average, makes up $27 \%$ of the total level of TAXI (40 kDa) (Fig. 3a), it would be expected to have a major influence on the total TAXI population, and probably on the xylanase inhibition activity. Nevertheless, based on activity measurements, Martonvasari-17 has a lower 'total' TAXI level (68 ppm) than Kirkpinar-17 (110 ppm) and Hereward (115 ppm). 


\section{Table 3 - Statistical analysis of identified wheat XI proteins.}

Spot no. ${ }^{\text {a) }}$ Protein name Mascot ${ }^{\text {b) }}$

$\% \operatorname{cov}^{\mathrm{c})}$

One-way ANOVA ${ }^{\text {d) }}$

Av. ratio ${ }^{\mathrm{e}}$

Log standard abundance profile ${ }^{\text {ff }}$

Protein score Total ion score Gl He Ki Kl-E Lo Ma

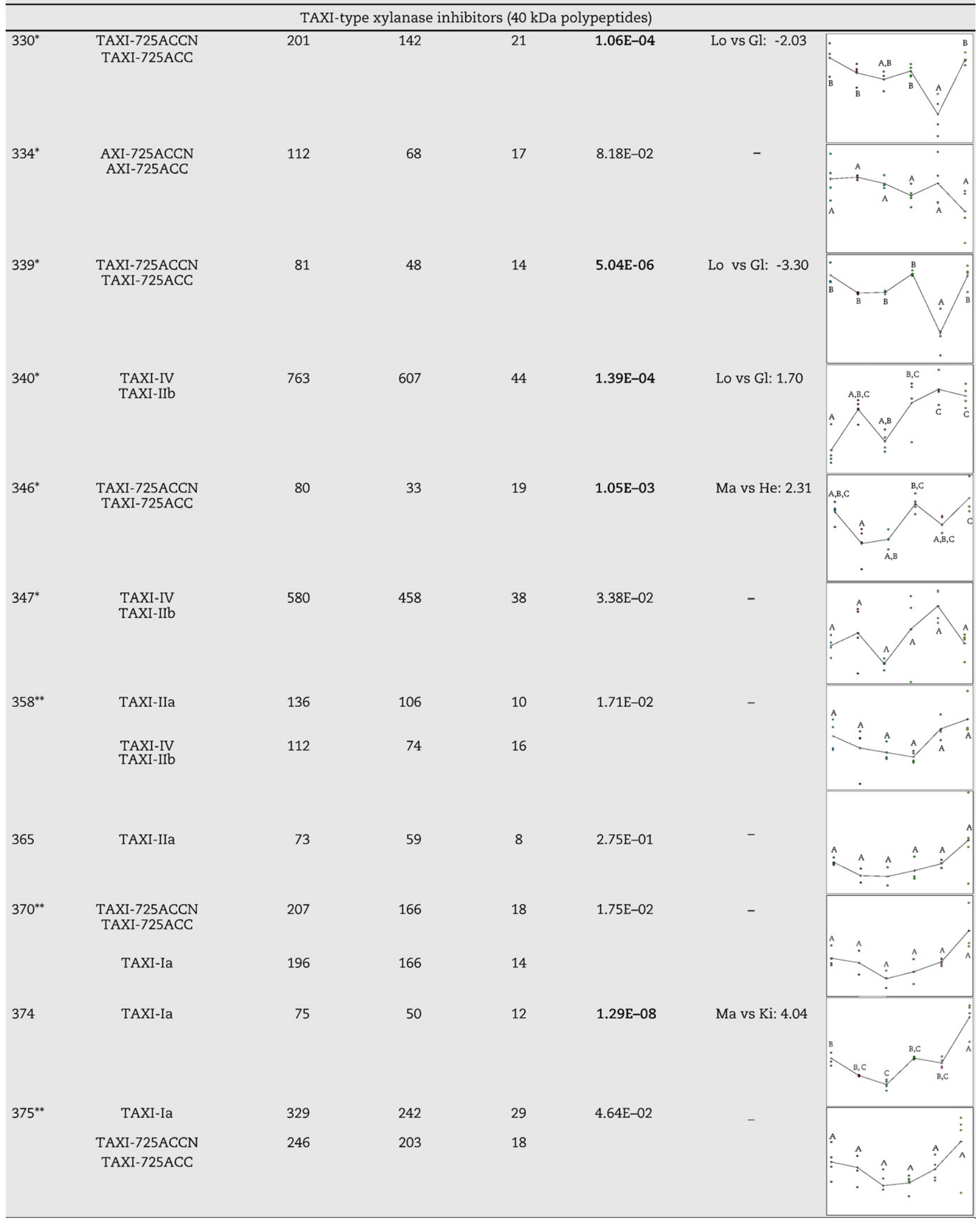




\section{Table 3 (continued)}

Spot no. ${ }^{\text {a) }}$ Protein name

Mascot $^{\mathrm{b})}$

Protein score Total ion score
Log standard abundance profile ${ }^{\mathrm{f}}$ Gl He Ki Kl-E Lo Ma

\begin{tabular}{|c|c|c|c|c|c|c|c|}
\hline \multicolumn{8}{|c|}{ TAXI-type xylanase inhibitors ( $30 \mathrm{kDa}$ polypeptides) } \\
\hline $606^{*}$ & $\begin{array}{l}\text { TAXI-725ACCN } \\
\text { TAXI-725ACC }\end{array}$ & 124 & 104 & 9 & $5.72 \mathrm{E}-07$ & Ma vs He: -2.66 & 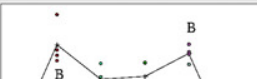 \\
\hline $636^{*}$ & $\begin{array}{l}\text { TAXI-725ACCN } \\
\text { TAXI-725ACC }\end{array}$ & 629 & 549 & 25 & $1.92 \mathrm{E}-08$ & Lo vs Kl-E: -3.66 & \\
\hline $661^{*}$ & $\begin{array}{l}\text { TAXI-IV } \\
\text { TAXI-IIb }\end{array}$ & 72 & 2 & 15 & $2.59 \mathrm{E}-07$ & He vs Gl: 3.30 & \\
\hline $669^{*}$ & $\begin{array}{l}\text { TAXI-725ACCN } \\
\text { TAXI-725ACC }\end{array}$ & 441 & 361 & 25 & 1.19E-05 & Lo vs He: -2.01 & \\
\hline $670^{* *}$ & TAXI-Ia & 150 & 99 & 18 & $2.31 \mathrm{E}-04$ & Ma vs He: -2.40 & . \\
\hline & $\begin{array}{l}\text { TAXI-725ACCN } \\
\text { TAXI-725ACC }\end{array}$ & 120 & 99 & 12 & & & $\begin{array}{l}\vdots \\
:\end{array}$ \\
\hline $671^{* *}$ & TAXI-Ia & 332 & 276 & 18 & $7.55 \mathrm{E}-05$ & Ma vs Kl-E: -3.06 & \\
\hline & $\begin{array}{l}\text { TAXI-725ACCN } \\
\text { TAXI-725ACC }\end{array}$ & 277 & 254 & 12 & & & \\
\hline $704^{* *}$ & TAXI-Ia & 191 & 148 & 16 & $8.86 \mathrm{E}-06$ & Ma vs Ki: -1.80 & \\
\hline & $\begin{array}{l}\text { TAXI-725ACCN } \\
\text { TAXI-725ACC }\end{array}$ & 137 & 115 & 12 & & & A $\dot{A}^{-} \dot{\mathrm{A}}$ \\
\hline
\end{tabular}

XIP-type xylanase inhibitors

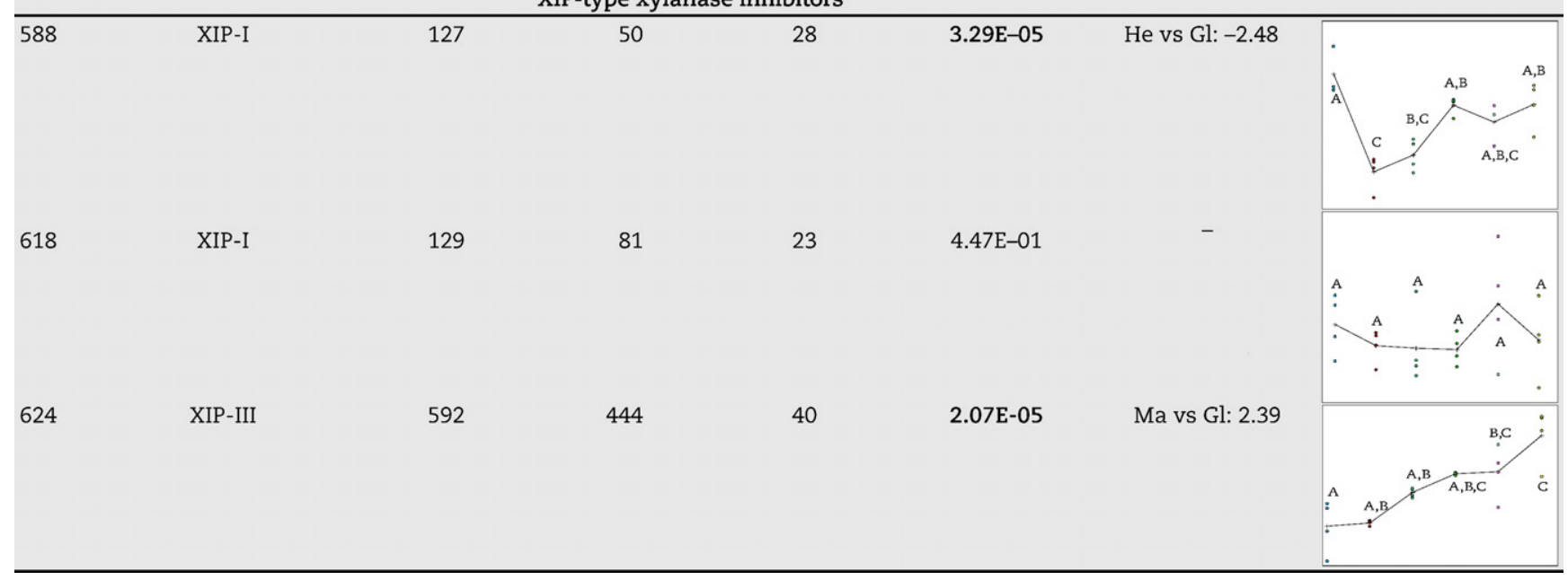




\section{Table 3 (continued)}

Spot no. a) Protein name

Mascot $^{\text {b) }}$

$\left.\% \operatorname{cov}^{c}{ }^{c}\right)$

One-way ANOVA ${ }^{\mathrm{d})}$

Av. ratio ${ }^{\mathrm{e}}$

Log standard abundance profile ${ }^{f}$

Protein score Total ion score Gl He Ki Kl-E Lo Ma

\begin{tabular}{|c|c|c|c|c|c|c|c|}
\hline & & & type & ibito & & & \\
\hline 635 & XIP-I & 245 & 85 & 48 & $1.38 \mathrm{E}-03$ & Ma vs Lo: -2.98 & \\
\hline 637 & XIP-I & 164 & 71 & 34 & $7.64 \mathrm{E}-07$ & Kl-E vs Gl: -2.40 & \\
\hline 639 & XIP-I & 186 & 99 & 37 & $2.30 \mathrm{E}-08$ & Ma vs Lo: -3.13 & \\
\hline 642 & XIP-I & 26 & - & 22 & $1.23 \mathrm{E}-05$ & Ma vs Kl-E: -2.58 & \\
\hline 643 & XIP-I & 759 & 566 & 59 & $1.97 \mathrm{E}-05$ & Ma vs Kl-E: -2.46 & \\
\hline 659 & XIP-III & 491 & 277 & 52 & $2.36 \mathrm{E}-08$ & Ma vs Gl: 5.03 & \\
\hline 674 & XIP-I & 597 & 457 & 44 & $5.75 \mathrm{E}-05$ & Kl-E vs Gl: 2.41 & \\
\hline 676 & XIP-I & 109 & 49 & 22 & $1.53 \mathrm{E}-02$ & Ma vs Lo: -5.30 & 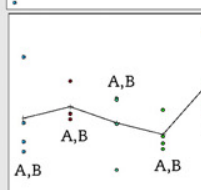 \\
\hline 677 & XIP-I & 848 & 652 & 59 & $5.04 \mathrm{E}-04$ & Ma vs $\mathrm{Kl}-\mathrm{E}:-2.78$ & \\
\hline 678 & XIP -I & 324 & 223 & 40 & $5.56 \mathrm{E}-05$ & Ma vs Lo: -2.51 & \\
\hline
\end{tabular}




\section{Table 3 (continued)}

\begin{tabular}{|c|c|c|c|c|c|c|c|}
\hline \multirow[t]{2}{*}{ Spot no. ${ }^{\text {a) }}$} & \multirow[t]{2}{*}{ Protein name } & \multicolumn{2}{|c|}{ Mascot $^{\text {b) }}$} & \multirow[t]{2}{*}{$\% \operatorname{cov}^{c)}$} & \multirow[t]{2}{*}{ One-way ANOVA $^{\text {d) }}$} & \multirow[t]{2}{*}{ Av. ratio $\left.{ }^{e}\right)$} & \multirow{2}{*}{$\begin{array}{l}\text { Log standard } \\
\text { abundance profile } \\
\text { Gl He Ki Kl-E Lo Ma }\end{array}$} \\
\hline & & Protein score & Total ion score & & & & \\
\hline \multicolumn{8}{|c|}{ XIP-type xylanase inhibitors } \\
\hline 679 & XIP-I & 115 & 25 & 28 & 3.73E-05 & Ma vs Kl-E: -2.66 & \\
\hline 689 & XIP-III & 274 & 164 & 38 & $4.06 \mathrm{E}-03$ & Kl-E vs He: 1.79 & \\
\hline 698 & XIP-I & 286 & 210 & 31 & 1.19E-02 & Lo vs He: 3.01 & \\
\hline 700 & XIP-III & 834 & 648 & 45 & $2.81 \mathrm{E}-08$ & Ma vs He: 3.77 & \\
\hline 706 & XIP-I & 359 & 261 & 31 & $3.32 \mathrm{E}-01$ & - & $\cdot$ \\
\hline 709 & XIP-I & 535 & 428 & 34 & $1.99 \mathrm{E}-04$ & Lo vs Ki: -2.38 & \\
\hline $711^{* *}$ & XIP-R2 & 267 & 197 & 23 & $3.26 \mathrm{E}-04$ & Lo vs $\mathrm{Ki}:-1.90$ & \\
\hline & XIP-I & 212 & 151 & 27 & & & $\dot{A}, \mathrm{~B}, \mathrm{~A}, \mathrm{~B}$ \\
\hline 713 & XIP -I & 828 & 632 & 59 & $2.09 \mathrm{E}-02$ & - & \\
\hline 714 & XIP-I & 142 & 90 & 28 & 5.80E-06 & Ma vs Ki: -2.42 & \\
\hline 716 & XIP-I & 171 & 96 & 27 & $1.05 \mathrm{E}-04$ & Lo vs Ki: -2.37 & \\
\hline & & & & & & & $\begin{array}{l}\stackrel{\mathrm{A}, \mathrm{B}, \mathrm{C}}{\mathrm{C}} \dot{\mathrm{C}}^{\mathrm{B}} \\
: \mathrm{A}, \mathrm{B}, \mathrm{C}\end{array}$ \\
\hline
\end{tabular}




\section{Table 3 (continued)}

\begin{tabular}{|c|c|c|c|c|c|c|c|}
\hline \multirow[t]{2}{*}{ Spot no. ${ }^{\text {a) }}$} & \multirow[t]{2}{*}{ Protein name } & \multicolumn{2}{|c|}{ Mascot ${ }^{b)}$} & \multirow[t]{2}{*}{$\left.\% \operatorname{cov}^{c}{ }^{c}\right)$} & \multirow[t]{2}{*}{ One-way ANOVA ${ }^{\text {d) }}$} & \multirow[t]{2}{*}{ Av. ratio ${ }^{\mathrm{e}}$} & \multirow{2}{*}{$\begin{array}{c}\text { Log standard } \\
\text { abundance profile } \\
\text { Gl He Ki Kl-E Lo Ma }\end{array}$} \\
\hline & & Protein score & Total ion score & & & & \\
\hline \multicolumn{8}{|c|}{ XIP-type xylanase inhibitors } \\
\hline 717 & XIP-I & 705 & 546 & 52 & $2.06 \mathrm{E}-08$ & Ma vs Ki: -2.52 & \\
\hline 720 & XIP-I & 266 & 191 & 27 & $2.80 \mathrm{E}-04$ & Ki vs Lo: -2.79 & \\
\hline 722 & XIP-I & 954 & 755 & 59 & $1.74 \mathrm{E}-02$ & - & \\
\hline 882 & XIP-I & 406 & 330 & 36 & $2.15 \mathrm{E}-04$ & $\begin{array}{l}\text { Ma vs Ki: } 2.00 \\
\text { Ma vs Kl-E: } 2.00\end{array}$ & \\
\hline \multicolumn{8}{|c|}{ TLXI-type xylanase inhibitors } \\
\hline 1017 & TLXI & 140 & 42 & 37 & $4.24 \mathrm{E}-11$ & Kl-E vs He: 4.56 & \\
\hline 1049 & TLXI & 369 & 305 & 25 & $3.31 \mathrm{E}-08$ & Ki vs He: 3.20 & \\
\hline 1055 & TLXI & 134 & 70 & 32 & $1.44 \mathrm{E}-06$ & Lo vs Ki: -4.30 & \\
\hline
\end{tabular}

a) Spot numbers as indicated in Fig. 2. b) Mascot probability scores for the entire protein and for ions. c) \% Sequence coverage. d) One-way ANOVA (as calculated in DeCyder EDA module) P-values $<0.01$ (bold) are considered significant. e) Average ratios ( $<<0.01$ in multiple comparison test) of largest difference in protein abundance between the cultivars. f) Log standard abundance profiles, generated in DeCyder EDA [ $x$-axis (from left to right): cultivars Glenlea, Hereward, Kirkpinar-79, Klein-Estrella, Lona, Martonvasari-17; $y$-axis: log standard abundance relative to the internal standard]. Significant $(P<0.01$, multiple comparison test) differences in protein abundance between the cultivars are indicated by different letters (A, B, C, D). Overlapping letters indicate no significant difference.

*Spot identified as one of two XI forms, which are highly similar sequence variants and therefore indistinguishable by our MS/MS results. ${ }^{* *}$ Spot containing a mixture of two XI genetic variants, as peptides for both were matched in MS/MS analysis.

High similarities in abundance profiles between the different cultivars are observed for spots 330 and 339, both identified as TAXI-725ACCN/TAXI-725ACC (Table 3). In addition, spots 340 and 347 (TAXI-IV/TAXI-IIb), on the one hand, and spots 346 (TAXI-725ACCN/TAXI-725ACC) and 374 (TAXI-Ia), on the other hand, are characterized by highly comparable abundance patterns among the six cultivars. A schematic PCA plot (Fig. 1b) of the identified proteins gives a rough estimate of the relationship between different proteins within a cluster and between individual proteins and wheat cultivars, represented by their replicate spot maps (Fig. 1a).
For 5 out of 7 identified $30 \mathrm{kDa}$ forms of TAXI-type XIs no reliable quantitative statements can be made, as they overlap with non-XI proteins. Average ratios $(P<0.01)$ between the cultivars range between 1.6 and 3.7 for spots 636 and 669, both identified as TAXI-725ACCN/TAXI-725ACC.

For XIP-type XIs, only 4 of the 27 identified spots do not show significant intercultivar differences. Overall XIP levels among the six cultivars were previously shown to vary by a factor 2.4 [21]. In this study, 5-fold differences in abundance between cultivars are demonstrated for some XIP (iso)forms while other forms show similar levels. The largest changes 
in abundance were found for spots 659 and 700, both identified as XIP-III. However, this variant only represents a minor proportion of the total XIP population. Highly similar abundance profiles are observed for the XIP-III spots, in particular between spots 624 and 659 (the more glycosylated forms) and spots 689 and 700 (the least glycosylated forms) (Table 3, Fig. 1b). In total, wheat cultivar Martonvasari-17 expresses the largest level of this genetic variant, while this is one of the cultivars with the lowest 'total' XIP levels.

A similar intercultivar variation was also demonstrated for the least (spots 709, 711, 716, 720 and spots 714 and 717), the more (spots 674, 677, 678 and 679) and the most glycosylated XIP-I forms (spots 635 and 639 and spots 637, 642 and 643), a distinction based on their increasing molecular masses (Table 3). They properly cluster in Fig. 1b. Spot 882, in turn, shows a high similarity to the abundance pattern of the least glycosylated XIPI forms. This spot is situated at a molecular mass of $\sim 25 \mathrm{kDa}$ and near neutral $\mathrm{pI}$ and is probably formed by cleaving of the Cterminal part of XIP-I, as peptides, matched in MS/MS, were situated close to the $\mathrm{N}$ - but none to the $\mathrm{C}$-terminus.

Three spots, identified as TLXI-type XIs, show remarkable changes in abundance between the six cultivars. Average ratios $(P<0.01)$ vary from $1.5-4.6$ (Table 3$)$, while the overall TLXI levels for the six cultivars vary by a factor 2.9 [21]. Correlations between the relative intensities of spots 1017 , 1049 and 1055 were found for the six cultivars (Table 3, Fig. 1b).

3.4.2. Similarities in intercultivar variation between XI forms of different classes

Not only within one class of XIs, but also between different TAXI, XIP and TLXI (iso)forms comparable intercultivar abundance patterns could be distinguished (Table 3, Fig. 1).
A similar pattern is observed for TLXI (spots 1017, 1049 and 1055) and XIP-III (spots 689 and 700), as well as for the $40 \mathrm{kDa}$ TAXI-725ACCN/TAXI-725ACC form (spots 330, 339), its cleaved counterpart (spots 636, 669) and several XIP-I forms (spots 709, $711,716)$. Thus, it can be hypothesized that the expression of some XI variants or the production of PTMs is co-regulated within the wheat grain. This, in turn, may well imply that several XI forms are involved in similar processes within the plant, possibly within plant defence.

\subsubsection{Similarities in intercultivar variation between XIs and} non-XI proteins

In a next step, correlations in relative levels of specific XI forms and non-XI proteins were sought for the six cultivars, focussing on the intercultivar variation in several other plant defence-related proteins.

In total, 16 chitinases ( $\mathrm{gi} \mid 2502681$, gi|75309544, gi|75262903, gi|62465514, gi|116316), 11 peroxidases (gi|157830301, gi| 22001285), 4 thaumatin-like proteins (TLP-7) (gi|14164981), 2 grain softness proteins ( $g i \mid 60652224), 2$ secretory proteins ( $g i \mid$ 5669008), 2 pathogenesis-related 1.1 proteins (gi|3702663), a small Ras-related GTP-binding protein (gi|16903082) and a wheatwin-2 precursor protein (gi|34925032) were identified (Table S6). Pattern analysis only revealed remarkable similarities between some XIs and chitinases. The positions of the different chitinase (iso)forms in the complex pattern of wheat seed proteins are indicated in Fig. 5. Based on their abundance profiles among the different cultivars, 3 groups of chitinases can be distinguished (Fig. 6). For each group, relative levels for the six cultivars similar to some XI forms were found, as shown in Figs. $1 \mathrm{~b}$ and 7. Group I, consisting of class I chitinases (spots 570, 571, 586 and 587), and TAXI-725ACCN/TAXI-725ACC/TAXI-

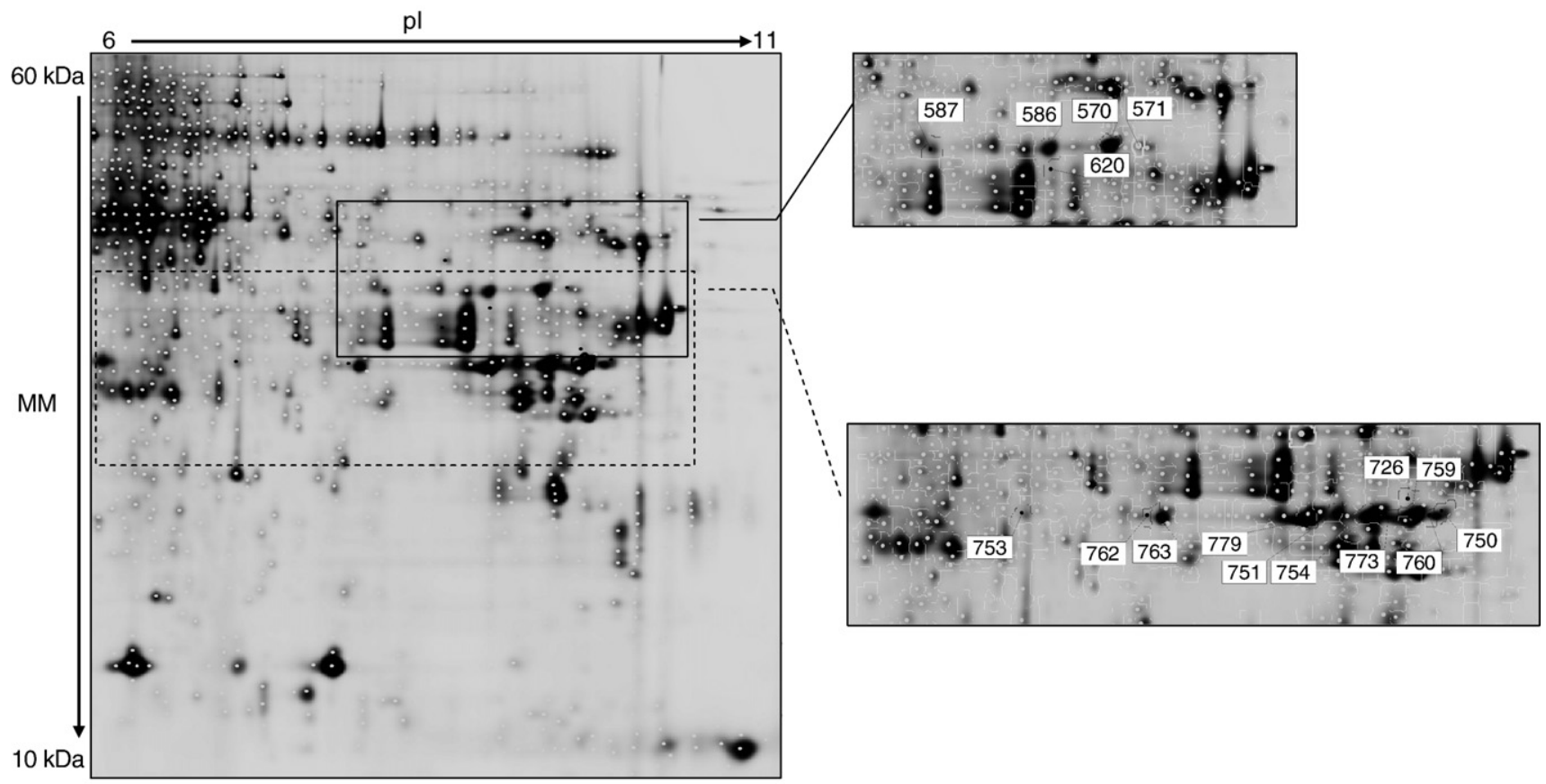

Fig. 6 - Positions of identified chitinases (spot IDs are indicated) within a representative spot map of wheat seed proteins (cultivar Klein-Estrella, Cy-5). Three groups are formed based on their abundance profiles (see Fig. 7): group I: spots 570, 571, 586, 587; group II: spots 726, 750, 759, 760, 773 and group III: spots 753, 762, 763, 779. 

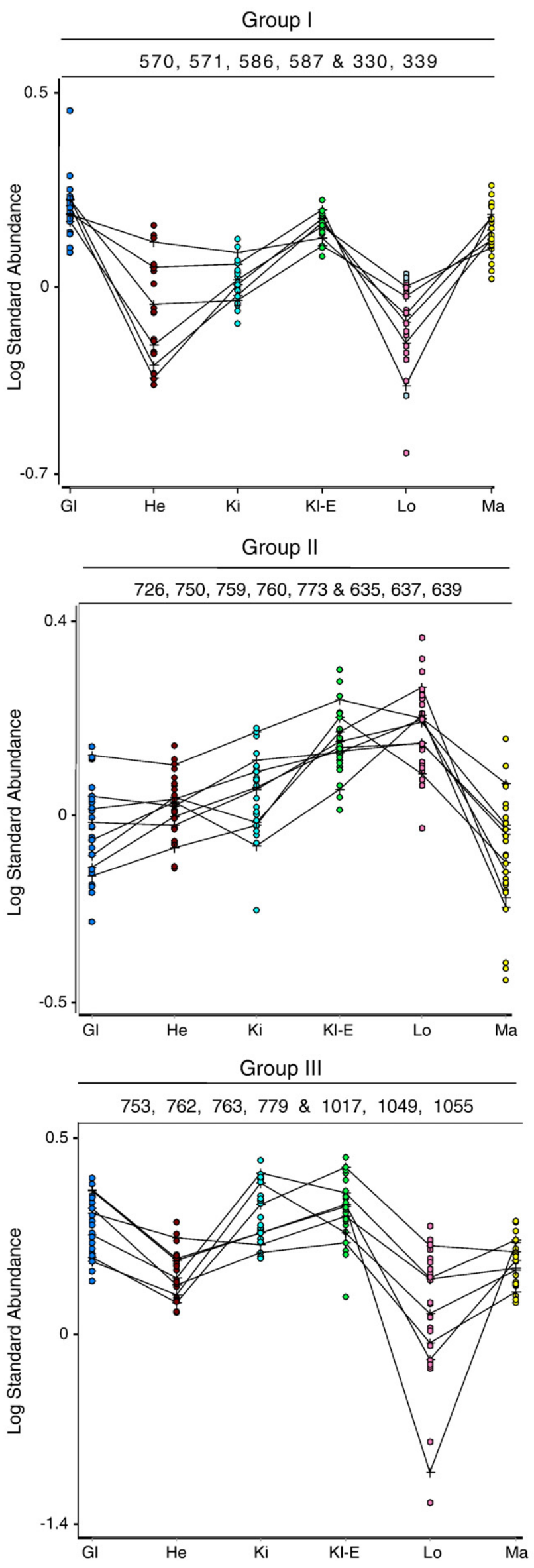

Ia (spots 330 and 339) show comparable patterns. Similarities in abundance were also found for group II, belonging to class II chitinases (spots 726, 750, 759, 760 and 773), and three XIP-I spots $(635,637$ and 639). Group III (spots 753, 762, 763 and 779) also consists of class II chitinases which are characterized by analogous abundance profiles, in particular to TLXI (spots 1017, 1049 and 1055).

\section{Discussion}

In this study, we have used the 2D-DIGE approach coupled to multivariate statistical analysis for the quantitative analysis of the large polymorphic families of the three currently known classes of XIs in six wheat cultivars and for discovery of promising correlated proteins.

Homology based peptide analysis led to the identification of 48 XI protein forms, of which 18 TAXI- (11 unprocessed and 7 cleaved forms), 27 XIP-, and 3 TLXI-type XIs. The high multiplicity of spots as well as their position within the 2D-gel corresponded remarkably well with our previous findings for affinity-purified XIs. An additional genetic variant, XIP-R2, for which it was not known if expression actually occurred in wheat grains, was identified, albeit present in very low levels. Earlier, it was stated that this isoform most likely resides in wheat plant parts other than the caryopsis, or occurs under stress conditions. It exerts a xylanase specificity distinct from that of XIP-I and XIP-III, as it previously escaped entrapment by the A. niger xylanase affinity column during XIP purification [11].

Based on our experimental data, 35 of the 48 identified XI spots were shown to display significantly different abundances among the six cultivars. Ratios $(P<0.01)$ of TAXI-, XIPand TLXI-type XIs ranged between 1.5-4.0, 1.4-5.3 and 1.5-4.6, respectively. Thus, the obtained differences in the relative levels of the XI (iso)forms are more pronounced than the overall intercultivar variability in TAXI, XIP and TLXI, measured previously (factor 2.4, 2.4 and 2.9, respectively) [21].

The relative contribution of each TAXI, XIP or TLXI (iso)form to the total level of TAXI, XIP and TLXI-type XIs, respectively, was determined. Overall, it can be stated that the total level of TAXI (40 kDa) proteins cannot be attributed to a single member of the large TAXI-gene family. In contrast, multiple TAXI isoforms (TAXI-725ACCN/725ACC, TAXI-Ia, TAXI-IIa, TAXI-IV/ TAXI-IIb) occur in considerable quantities under healthy conditions. The opposite was found for XIP-type proteins. On average, XIP-I makes up approximately $92 \%$ of the 'total' XIP level. These results imply that the development by genetic engineering of TAXI-free or low-TAXI wheat cultivars, attractive for industrial applications in which microbial, inhibitorsensitive xylanases are added as processing-aids, would be a

Fig. 7 - Log standard abundance profiles of XI and chitinase spots (designated by their master spot IDs, Figs. 2 and 5) among the six cultivars, indicating the similarities in relative levels between some XI (iso)forms and these PR-proteins. Group I: class I chitinases and TAXI-725ACCN/725ACC/Ia; Group II: class II chitinases and XIP-I; Group III: class II chitinases and TLXI. 
very difficult task, whereas this would be more feasible for the XIP counterparts.

Comparison of the log standard abundance profiles of the multiple TAXI ( $30 \mathrm{kDa}$ and $40 \mathrm{kDa}$ ) forms with the differences in 'total' TAXI levels (Table 1) [21], allows to conclude that a combination of all the different forms is presumably decisive for the 'total' XI activity. However, the highest correlations $(P<0.05)$ were found between the overall TAXI level and TAXI-725ACCN/ TAXI-725ACC (30 and $40 \mathrm{kDa}$ ). Since this genetic variant does not make up the largest part of the total TAXI population (Fig. 3a) this would mean that TAXI-725ACCN/TAXI-725ACC can be distinguished by a higher specific activity than the other genetic variants. As 6 out of the 7 TAXI $30 \mathrm{kDa}$ spots were identified as TAXI-725ACCN/TAXI-725ACC, it could be suggested that this is the predominant form after cleaving. Previous results had shown that mature wheat grains contain approximately equal levels of the processed and the unprocessed forms [21]. Thus, another explanation for the observed correlation may be that the processed form of TAXI-725ACCN/TAXI-725ACC has a more profound influence on the total XI activity.

The intercultivar variation in 'total' XIP levels [21] correlates $(P<0.05)$ with the more and most glycosylated XIP-I forms. However, they do not represent the major part of the 'total' XIP population (Fig. 3b). It can be hypothesized that a higher degree of glycosylation increases the XI activity. It has been suggested that glycosylation is involved in stabilizing the activity of native XIP-I [5].

The observed pattern of spot 1017, the most glycosylated form of TLXI which contributes most to the 'total' TLXI population (Fig. 3c), corresponds $(P<0.05)$ well to the intercultivar variation measured for the 'total' pool of TLXI-type XIs [21].

Several XI (iso)forms, either from one XI class or from different XI classes, show highly similar abundance patterns among the six wheat cultivars. This implies that the regulation of the expression or the formation of PTMs of several XI forms is strongly linked within the wheat grain, and hence, that they serve a common goal within the plant, probably in plant defence. The XI (iso)forms showing distinct patterns may have a more specialized role in the protection of the plant. It has been demonstrated that XIs can largely differ in their specificities towards xylanases, e.g. for TAXI-Ia and TAXI-IIa and XIP-I and XIP-R1. This hypothesis is in line with transcriptomic studies on TAXI- and XIP-type XIs. Some XI (iso)forms are considered to act as a basal pre-existing defence mechanism against pathogens during seed development and germination, while the transcription of other XI genes is governed by (a)biotic stress signals, albeit depending on the type and site of infestation [8-10].

Based on the above and the current work, we can suggest that several XI genes within the XI gene families, such as Xip-I, Taxi-Ia and Tlxi, are constitutively expressed under various physiological conditions, while a higher accumulation of e.g. Xip-III and Xip-R2 transcripts may be induced under more restricted conditions (e.g. stress, pathogen attack) [10]. Moreover, XI genes, which were not found to be expressed in healthy grains, such as Taxi-III or Xip-R1, may be exclusively regulated in response to specific pathogens or stress conditions. This is consistent with our finding that XIP-R2 is present in wheat grains in the absence of XIP-R1. Takahashi-Ando et al. [10] already stated that Xip-R2 is predominantly expressed in uninfected wheat tissues, while Xip-R1 transcripts are mainly induced after attack with the pathogen Erysiphe graminis.

Besides a differential constitutive and induced expression of XI isoforms, the specific activity or location of these plant defence-related proteins may be regulated on a second level by post-translational modifications. While the largest part of the XIP-type XIs in this study have no or only a moderate level of sugar residues attached, the majority of the TLXI-type proteins is highly glycosylated. Glycosylation affects protein folding, localization and trafficking, protein solubility, antigenicity, biological activity, as well as cell-cell interactions [29]. Moreover, stabilization of proteins by the glycan chains is proportionate to the number of attached sugar residues [30]. Thus, a variable degree of glycosylation may well provide an additional means to modulate the properties of XIP- and TLXItype proteins. This corresponds with the above made observation on higher specific activity of glycosylated XI forms.

As a considerable variation exists in the basal and/or induced levels of the different (iso)forms between the six cultivars, this would mean that their degree of resistance against pathogenic attack probably varies according to their unique XI population. As an example, cultivars Lona and Glenlea contain relatively high and low levels, respectively, of TAXI-IV/IIb, known to be inducible by both Fusarium graminearum and E. graminis infection. XIP-I, which was only enhanced in leaves in response to $E$. graminis and wounding, is highly represented in the cultivar Klein-Estrella. However, no information is available on the pathogen- or stress-induced expression of e.g. Taxi-725ACCN, Xip-III or Tlxi. Lots of research still need to be done to determine to which extent all different XI genes are induced depending on the (a)biotic signal or site of attack and whether they are involved in stress-related signaling pathways, as was partly done for several Xip genes in rice [31,32].

Additional evidence for the role of XIs in plant defence was provided by a comparison with other identified PR-proteins. Correlations were found between the levels of several basic class I and class II chitinase (iso)forms and several forms of TAXI, XIP and TLXI. Chitinases are considered to be plant defence-related proteins as they protect against fungal pathogens by degrading chitin, a major component of the cell walls of fungi [33]. Plant chitinases are proteins of $25-35 \mathrm{kDa}$, of which many are located in the apoplast, similar to XIP-R1 [10]. The presence of signal peptides in all the members of the three classes of XIs equally points to their extracellular localization. Similar to what is described for XIs, several chitinases are constitutively expressed, while some are induced by pathogen attack and treatment with elicitors and abiotic factors, such as plant hormones [33]. The similarity in abundance between some XI (iso)forms and several chitinases may well point to the co-regulation of their expression or formation of PTMs within the wheat grain and gives an indication of their cooperative action in plant protection. A synergetic action in the inhibition of fungal growth was previously observed for chitinases and $\beta$-1,3-glucanases [33].

In conclusion, the work presented here shows that differences in XI levels between wheat cultivars can be attributed to various individual differences in the multiple (iso)forms, in which they occur. Highly distinctive as well as correlated (expression) levels of XI forms within one class and between different classes of XIs was found among the six wheat 
cultivars. Furthermore, we suggested a coordinated expression or formation of PTMs for XIs and chitinases. Cultivar-dependent variations in the constitutive and/or induced levels of the large polymorphic XI families probably affect their susceptibility to pathogen infection or stress conditions. However, it remains to be investigated in detail how different XI (iso)forms are regulated and to which extent they individually may contribute to plant defence.

\section{Acknowledgements}

The authors would like to thank Gert Raedschelders (Laboratory of Gene Technology, K.U. Leuven) for fruitful discussions.

The authors acknowledge the 'Instituut voor de aanmoediging van Innovatie door Wetenschap en Technologie in Vlaanderen' (I.W.T., Brussels, Belgium) for their financial support. Kurt Gebruers and Sebastien Carpentier are postdoctoral fellows of the Fonds voor Wetenschappelijk OnderzoekVlaanderen (FWO-Vlaanderen, Brussels, Belgium). This study was in part carried out in the framework of research project GOA/03/10 financed by the K.U. Leuven Research Fund and the Methusalem programme 'Food for the Future' at the K.U. Leuven.

\section{Appendix A. Supplementary data}

Supplementary data associated with this article can be found, in the online version, at doi:10.1016/j.jprot.2009.02.003.

\section{R E F E R E N C E S}

[1] Wanjiru WM, Kang ZS, Buchenauer H. Importance of cell wall degrading enzymes produced by Fusarium graminearum during infection of wheat heads. Eur J Plant Pathol 2002;108:803-10.

[2] Debyser W, Derdelinckx G, Delcour JA. Arabinoxylan solubilization and inhibition of the barley malt xylanolytic system by wheat during mashing with wheat wholemeal adjunct: evidence for a new class of enzyme inhibitors in wheat. J Am Soc Brew Chem 1997;55:153-6.

[3] Fierens K, Brijs K, Courtin CM, Gebruers K, Goesaert H, Raedschelders G, et al. Molecular identification of wheat endoxylanase inhibitor TAXI-I-1, member of a new class of plant proteins. FEBS Letters 2003;540:259-63.

[4] McLauchlan WR, Garcia-Conesa MT, Williamson G, Roza M, Ravestein P, Maat J. A novel class of protein from wheat which inhibits xylanases. Biochem J 1999;338:441-6.

[5] Elliott GO, Hughes RK, Juge N, Kroon PA, Williamson G. Functional identification of the cDNA coding for a wheat endo-1,4-beta-D-xylanase inhibitor. FEBS Letters 2002;519:66-70.

[6] Fierens E, Rombouts S, Gebruers K, Goesaert H, Brijs K, Beaugrand J, et al. TLXI, a novel type of xylanase inhibitor from wheat (Triticum aestivum) belonging to the thaumatin family. Biochem J 2007;403:583-91.

[7] Gebruers K, Debyser W, Goesaert H, Proost P, Van Damme J, Delcour JA. Triticum aestivum L. endoxylanase inhibitor (TAXI) consists of two inhibitors, TAXI I and TAXI II, with different specificities. Biochem J 2001;353:239-44.
[8] Igawa T, Ochiai-Fukuda T, Takahashi-Ando N, Ohsato S, Shibata T, Yamaguchi I, et al. New TAXI-type xylanase inhibitor genes are inducible by pathogens and wounding in hexaploid wheat. Plant Cell Physiol 2004;45:1347-60.

[9] Igawa T, Tokai T, Kudo T, Yamaguchi I, Kimura M. A wheat xylanase inhibitor gene, Xip-I, but not Taxi-I, is significantly induced by biotic and abiotic signals that trigger plant defense. Biosci Biotechnol Biochem 2005;69:1058-63.

[10] Takahashi-Ando N, Inaba M, Ohsato S, Igawa T, Usami R, Chimera M. Identification of multiple highly similar XIP-type xylanase inhibitor genes in hexaploid wheat. Biochem Biophys Res Commun 2007;360:880-4.

[11] Croes E, Gebruers K, Robben J, Noben JP, Samyn B, Debyser G, et al. Variability of polymorphic families of three types of xylanase inhibitors in the wheat grain proteome. Proteomics 2008;8:1692-705.

[12] Misas-Villamil JC, van der Hoorn RAL. Enzyme-inhibitor interactions at the plant-pathogen interface. Curr Opin Plant Biol 2008;11:380-8.

[13] Stahl EA, Bishop JG. Plant-pathogen arms races at the molecular level. Curr Opin Plant Biol 2000;3:299-304.

[14] D'Ovidio R, Mattei B, Roberti S, Bellincampi D. Polygalacturonases, polygalacturonase-inhibiting proteins and pectic oligomers in plant-pathogen interactions. Biochim Biophys Acta 2004;1696:237-44.

[15] Juge N, Svensson B. Proteinaceous inhibitors of carbohydrate-active enzymes in cereals: implication in agriculture, cereal processing and nutrition. J Sci Food Agric 2006;86:1573-86.

[16] Trogh I, Sorensen JF, Courtin CM, Delcour JA. Impact of inhibition sensitivity on endoxylanase functionality in wheat flour breadmaking. J Agric Food Chem 2004;52:4296-302.

[17] Frederix SA, Courtin CM, Delcour JA. Substrate selectivity and inhibitor sensitivity affect xylanase functionality in wheat flour gluten-starch separation. J Cereal Sci 2004;40:41-9.

[18] Courtin CM, Gys W, Gebruers K, Delcour JA. Evidence for the involvement of arabinoxylan and xylanases in refrigerated dough syruping. J Agric Food Chem 2005;53:7623-9.

[19] Bonnin E, Daviet S, Gebruers K, Delcour JA, Goldson A, Juge N, et al. Variation in the levels of the different xylanase inhibitors in grain and flour of 20 French wheat cultivars. J Cereal Sci 2005;41:375-9.

[20] Dornez E, Joye IJ, Gebruers K, Lenartz J, Massaux C, Bodson B, et al. Insight into variability of apparent endoxylanase and endoxylanase inhibitor levels in wheat kernels. J Sci Food Agric 2006;86:1610-7.

[21] Croes E, Gebruers K, Luyten N, Delcour JA, Courtin CM. Immunoblot quantification of three classes of proteinaceous xylanase inhibitors in different wheat cultivars and milling fractions. J Agric Food Chem 2009;57:1029-35.

[22] Laemmli UK. Cleavage of structural proteins during the assembly of the head of bacteriophage T4. Nature 1970;227:680-5.

[23] Witters E, Laukens K, Deckers P, Van Dongen W, Esmans E, Van Onckelen H. Fast liquid chromatography coupled to electrospray tandem mass spectrometry peptide sequencing for cross-species protein identification. Rapid Commun Mass Spectrom 2003;17:2188-94.

[24] Carpentier SC, Panis B, Vertommen A, Swennen R, et al. Proteome analysis of non-model plants: a challenging but powerful approach. Mass Spectrom Rev 2008;27:354-77.

[25] Perkins DN, Pappin DJC, Creasy DM, Cottrell JS. Probability-based protein identification by searching sequence databases using mass spectrometry data. Electrophoresis 1999;20:3551-67.

[26] Rice P, Longden I, Bleasby A. EMBOSS: the European molecular biology open software suite. Trends Genet 2000;16:276-7.

[27] Raedschelders G, Fierens K, Sansen S, Rombouts S, Gebruers $\mathrm{K}$, Robben J, et al. Molecular identification of wheat 
endoxylanase inhibitor TAXI-II and the determinants of its inhibition specificity. Biochem Biophys Res Commun 2005;335:512-22.

[28] Rombauts S, Florquin K, Lescot M, Marchal K, Rouze P, Van de Peer Y. Computational approaches to identify promoters and cis-regulatory elements in plant genomes. Plant Physiol 2003;132:1162-76.

[29] Varki A. Biological roles of oligosaccharides - all of the theories are correct. Glycobiology 1993;3:97-130.

[30] Shental-Bechor D, Levy Y. Effect of glycosylation on protein folding: a close look at thermodynamic stabilization. PNAS 2008;105:8256-61.
[31] Tokunaga T, Esaka M. Induction of a novel XIP-type xylanase inhibitor by external ascorbic acid treatment and differential expression of XIP-family genes in rice. Plant Cell Physiol 2007;48:700-14.

[32] Tokunaga T, Miyata Y, Fujikawa Y, Esaka M. RNAi-mediated knockdown of the XIP-type endoxylanase inhibitor gene, OsXIP, has no effect on grain development and germination in rice. Plant Cell Physiol 2008;49:1122-7.

[33] Collinge DB, Kragh KM, Mikkelsen JD, Nielsen KK, Rasmussen U, Vad K. Plant chitinases. Plant J 1993;3:31-40. 